\title{
Accuracy assessment of SAR interferometry using the ERS-1
}

\author{
D. Carrasco, J. Alonso, A. Broquetas \\ Antennas, Microwaves and Radar Group \\ Department of Signal Theory and Communications \\ Universidad Politécnica de Cataluña \\ Gran Capitán s/n. Campus Norte UPC - Módulo D3. 08071 Barcelona (Spain) \\ Tel: +34 3 4016849,Fax: +3434017232, e-mail: diaz@voltor.upc.es
}

\begin{abstract}
A SAR raw data simulator and a SAR processor are used as a tool for performance assessment of SAR interferometry algorithms like the multi-baseline and multifrequency. Traditional algorithms have also been tested with real ERS-1 data and validated with a high accuracy reference DEM
\end{abstract}

\section{INTRODUCTION}

Obtaining digital elevation models (DEM) with interferometric techniques has become one of the major potential applications of SAR satellites. The performance assessment of SAR Interferometry (InSAR) is often difficult by its inherent complexity, the multiple sources of error (decorrelation, layover, etc.) and the lack of sufficiently accurate reference DEM over suitable regions. For this reasons, a raw data interferometric SAR simulator has been developed [1], which has allowed a detailed study of the InSAR algorithms performance. Given a reference DEM and a radar reflectivity map, the simulator obtains for a given sensor flight the hologram or raw-data. The raw-data is then processed with a phase preserving algorithm. Finally, the interferometric process is performed.

The simulator has been used to develop and improve the phase-unwrapping procedures, and to study the multibaseline and multibaseline approaches. Traditional single baseline techniques have also been tested with real ERS-1 data. The results have been validated through comparison with a high accuracy reference DEM. This allows the detection of unexpected errors as will be shown.

\section{THE SAR SIMULATOR}

The SAR raw data simulator is divided in two blocks. The first one, projects the ground scatterers over the slant-range plane and the second one generates the hologram or raw data.

The first block starting point are the satellite orbital parameters and a DEM and a dielectric characterization of the area under test. From the DEM. the Earth surface is modelled in facets. Each facet is assigned a reflection coefficient depending on its distance to the satellite, its roughness (which is modelled through the beamwidth of the radiation pattern) and its dielectric properties. Speckle is simulated by adding coherently nine facets (independent scatterers) within the same resolution cell. Each facet is obtained from the DEM adding a gaussian height which randomizes the reflectivity of the point scatterers and generates speckle. Finally, every resolution cell is projected over the slant range plane so that foreshortening, layover and shadowing phenomena appear naturally.

The second block of the simulator generates the raw data from the slant-range reflectivity map. The hologram can be generated by convolving the reflectivity map with the SAR impulse response (generated by a reflectivity delta). The calculation is implemented in the transformed Fourier domain. The main problem is that the SAR impulse response is range variant, but this can be solved by preprocessing the reflectivity map which can then be multiplied with the nonvariant SAR impulse response and then two-dimensional reverse Fourier transformed to obtain the final hologram.

Once we have the raw data, we can feed it into a phasepreserving SAR processor and obtain the image. The combination SAR processor-simulator is a very powerful tool which we have used to test new SAR interferometry algorithms.

\section{INTERFEROMETRY WITH SIMULATED DATA}

We have worked with the multibaseline and multifrequency approaches [2]. Both methods lie on the same principle: generate two interferograms with different fringe frequency, which will lead to an unambiguous phase unwrapping. Since the interferometric phase is proportional to the frequency and the baseline separation, by changing any of them we can modify the fringe frequency. In the multifrequency approach, two interferograms are generated at two different frecuencies (4 SAR images are needed). The multibaseline algorithm requires only three images and it may be applied to real ERS-1 data.

The SAR simulator has allowed us to test these procedures. As an example, the results of a simulation over an area in Catalonia with the multifrequency unwrapping algorithm are shown. In Fig. 1, one of the interferograms obtained from the processed SAR raw data is shown. The final DEM is presented in Fig. 2. The average absolute error is $9.1 \mathrm{~m}$ and the rms crror is $15.6 \mathrm{~m}$. Most errors are concentrated in the hilly area. 


\section{INTERFEROMETRY WITH REAL ERS-I DATA}

We have carried out the interferometric processing over a small area in Tarragona (Spain) very close to Ebro river. Our first aim was to validate the resulting DEM with a high accuracy reference DEM since a comparison with a topographic map could hide systematic errors and would not allow a real validation of the interferometric process.

The images were acquired on $12^{\text {th }}$ and $15^{\text {th }}$ september 1991 during the commissioning phase ( 3 day repeat cycle) and the baseline perpendicular component was $160 \mathrm{~m}$.

First of all, registration of the two images was performed in two stages. As a first step, a coarse registration was done through cross-correlation of the amplitude of two windows in each image. The amplitude was quantized to three levels in order to achieve a higher peak of the cross-correlation. Secondly, a fine registration was performed in the four corners of the image interpolating and shifting one image secking for a coherence maximum. The shift applied to the center of the image was interpolated from the values of the four corners.

Both images were filtered in the range direction since the spectrums of the two images are shifted and only the common band leads to the generation of fringes [3]. The spectral shift is calculated by spectrum cross-correlation of the two images. Then each image is filtered lcaving only the common frequency band. Notice that since the selected area is small and there is a significative average slope, we can carry out this process over the whole image without slopedependant segmentation. After the filtering, some spatial resolution is lost, but the coherence is higher. Range filtering lowers bandwidth a $15.7 \%$ and spatial resolution in the slant range direction lowers from $8.58 \mathrm{~m}$ to $9.93 \mathrm{~m}$.

Now the complex interferogram may be constructed by multiplicating one image by the complex conjugate of the other image. The phase will show the interferometric fringes. From the orbit data and the Earth coordinates of the

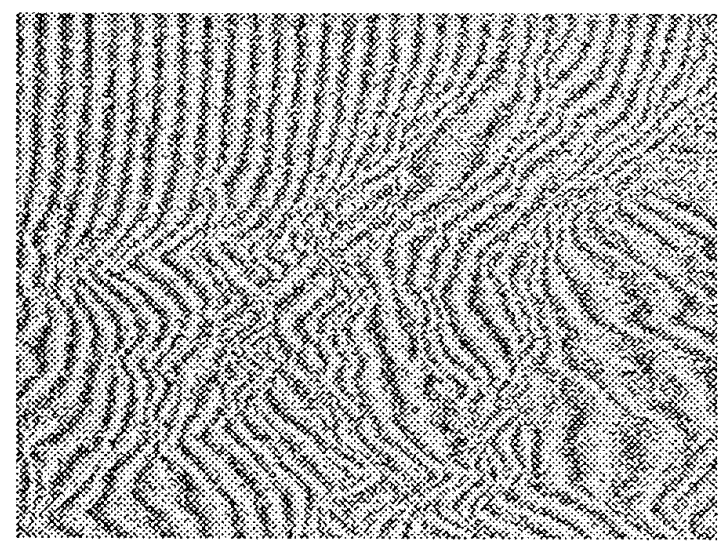

Fig. 1. Interferometric fringes from simulated SAR raw data image, we calculate for each pixel of the interferogram the distance to the satellite assuming a spherical Earth. This will allow us to substract the phase due not to the height variations but to the flat contribution of the ground.

The phase is then averaged with a pyramidal filter ( 9 pixels in azimuth by 5 pixels in range). Resolution is now $17.9 \mathrm{~m}$ in azimuth and $19.8 \mathrm{~m}$ in range. The filtered phase is shown in Fig. 3. It is very smooth and the phase unwrapping was not critical since the filtering reduced notably the noise level.

The phase unwrapping method is based in the residue theory [4]. Residues are joined in pairs through ghost lines which are not to be crossed when integrating the phase. Once the heights are known, we can project them over the ground.

The resulting DEM was compared to a high accuracy reference DEM which has $2 \mathrm{~m}$ rms height error and a $15 \mathrm{~m}$ by $15 \mathrm{~m}$ grid. We selcetcd three local maxima in the InSAR DEM and looked for their equivalents in the reference DEM. With this information, the high accuracy DEM was stretched and rotated so that it sized the SAR DEM. Then, the original heights in the SAR DEM were restored by adding a constant in order to achieve the save average height in both DEM. The absolute value of the difference between both DEM was computed and the result is shown in Fig. 4. Notice that the error is minimum in the image center and grows linearly in the azimuth direction. We have a linear component added to the original DEM. This is due to a spectral shift of the two SAR images in the azimuth direction which can be caused by a different Doppler centroid in the two images [2].

This problem was only detected by checking the obtained DEM with the reference DEM. This fact stresses the need of ground control points in the image of known height in order to correct systematic errors, as the one shown before, when no reference DEM is available.

Finally, the spectral shift in the azimuth was eliminated and the final DEM is shown in Fig. 5. The error between this

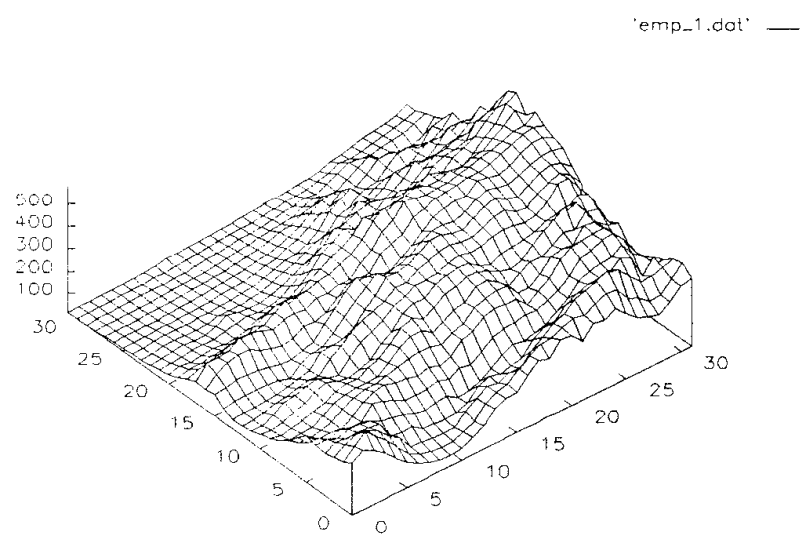

Fig. 2. DEM obtained by the multifrequency method. 
corrected DEM and de reference DEM is shown in Fig. 6. $70 \%$ of the points in the obtained DEM show an error under $10 \mathrm{~m}$ and the $95 \%$ is under $20 \mathrm{~m}$. The rms error is $10.36 \mathrm{~m}$ and the medium error is $8.27 \mathrm{~m}$.

\section{CONCLUSIONS}

A SAR simulator-processor has been presented as a tool for SAR interferometry algorithms test. Multifrequency results have been presented. Finally, it is shown how a reference DEM can help in detecting systematic errors. Ground control points should be used when it is not available.

\section{ACKNOWLEDGMENTS}

This work has been supported by the Com. Interministerial de Ciencia y Tecnología CICYT TIC 92-0645.

The authors wish to thank the ESA ERS-1 Fringe Working Group for providing the SAR images and the Institut Cartogràfic de Catalunya for supplying the reference DEM.

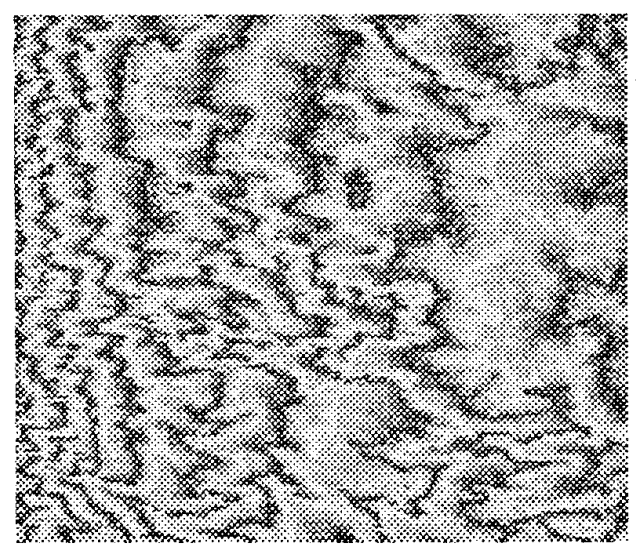

Fig. 3. Interferometric fringes filtered and ground corrected.

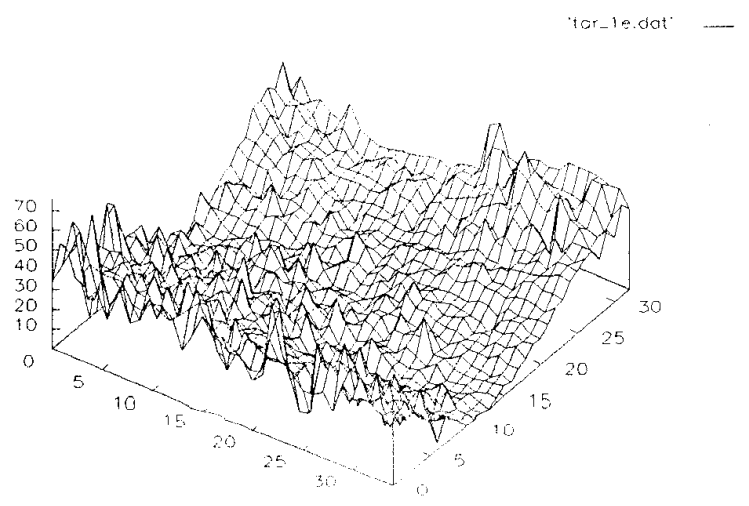

Fig. 4. Absolute error between the InSAR DEM and the reference DEM

\section{REFERENCES}

[1] G. Franceschetti, D. Riccio, "SARAS: a synthetic aperture radar (SAR) raw signal simulator", IEEE Trans. on Geoscience and Remote Sensing, vol 30, $\mathrm{n}^{\circ} 1$, pp. 110-122, Jan. 1992.

[2] J. Alonso, "Interferometría SAR mediante diversidad en frecuencia y en líneas de base", Proyecto Final de Carrera, ETS de Ingeniería de Telecomunicación de Barcelona, 1995.

[3] F. Gatelli et al., "The wavenumber shift in SAR interferometry", IEEE Trans. on Geoscience and Remote Sensing, vol. 32, n 4 , pp. 855-864, July 1994.

[4] R. Goldstein, H. Zebker, C. Werner, "Satellite radar interferometry: two-dimensional phase unwrapping", Radio Science, vol. 23, no 4, pp. 713-720, July-August 1988.

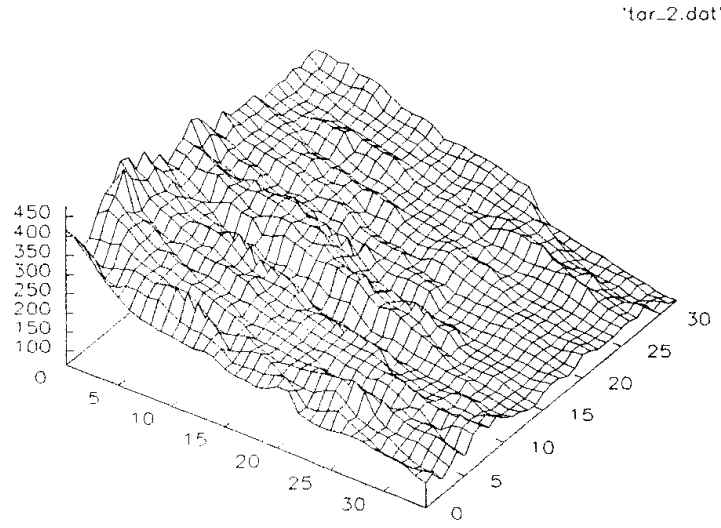

Fig. 5. Final DEM obtained.

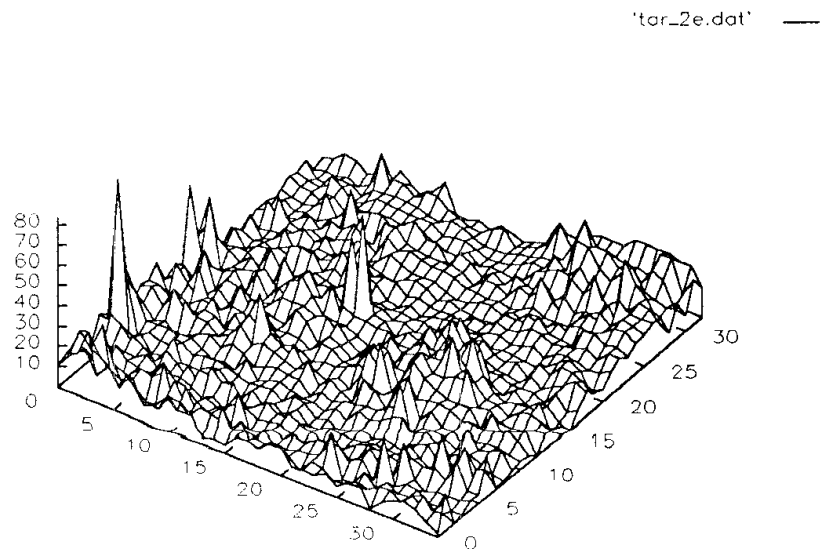

Fig. 6. Absolute crror between the final InSAR DEM and the reference DEM 